\title{
Community Health Workers delivering child health interventions: Evidence-base and key considerations
}

Tanya Doherty ${ }^{1,3}$, Nick Oliphant ${ }^{2}$ and David Sanders ${ }^{3}$

${ }^{1}$ South African Medical Research Council; ${ }^{2}$ UNICEF, New York; ${ }^{3}$ School of Public Health, University of the Western Cape

\section{Introduction}

This paper, presented as part of a workshop at the 2016 World Public Health Nutrition Conference, is one of a series of papers focused on 'magic bullets' versus community action for nutrition. This paper will describe the evidence base on the impact of CHW programmes for child health and discuss features of successful programmes. This is a timely issue as the World Health Organisation is currently leading a process to develop global guidance on community health worker programmes.

Despite the gains in reducing under-5 mortality globally, much progress is still needed, particularly in sub-Saharan Africa, the region with the highest under-5 mortality rate of 83 per 1000 live births in 2015. ${ }^{1}$ Although there have been increases in access to high impact interventions for children, by the end of the Millennium Development Goal (MDG) period, around $40 \%$ of children in the 75 countries with the highest under-5 mortality received oral rehydration solution for diarrhea and a similar proportion of infants under six months were currently being exclusively breastfed. ${ }^{2}$ Moreover, large equity gaps exist within countries in these coverage indicators and in outcomes ${ }^{3}$, particularly stunting which is concentrated among the poor, children living in rural areas, and those whose mothers have a low level of education. ${ }^{2}$

Community-based delivery platforms using community health workers (CHWs) have been implemented since the 1970s with increased focus following the Alma Ata Conference on primary health care. ${ }^{4}$ The early period of national implementation showed a mixed picture of success and failure. ${ }^{5}$ With the increased human resources for health requirements posed by the HIV/AIDS epidemic, pressure to meet the MDG and now SDG goals combined with the human resources for health crises in sub-Saharan Africa, ${ }^{6} \mathrm{CHW}$ programmes have re-emerged as a common strategy to increase access to health services particularly in rural and hard to reach areas. $^{7}$ 
Much of the scope of work of CHWs has been focused on child health, with some programmes having a broad scope including prevention and the promotion of interventions to support child health, whilst others have been specifically focused on nutrition or vertical programs for HIV, tuberculosis, and malaria. ${ }^{8-10}$ This paper does not focus specifically on community-based nutrition programmes, as a separate paper in this issue of WN does so (see Shrimpton and Shantha). There is now wide recognition that CHWs can effectively deliver many of the key maternal and child survival and nutrition interventions in the context of strong community trust and demand, mechanisms for motivation, and functioning systems for supplies, supportive supervision/mentoring and referrals. ${ }^{9,11}$ The evidence is strongest for the role of CHWs in newborn and maternal and young child health rather than nutrition specific outcomes (which have also received less attention until recently, except for growth monitoring which has not proven to be successful in several evaluations).

A key consideration in this era of renewed focus on community delivery platforms is whether their operations are truly integrated into comprehensive primary health care delivery or yet another vertically-driven 'magic bullet'. Governments and policy makers are grappling with these issues within the context of strong donor dependence and rapid policy change resulting in new interventions frequently being added, often stressfully, to the community delivery platform.

\section{The evidence for the impact of CHW programmes on child health}

Assessing impact of CHW programmes at scale (see below) is challenging due to the lack of funding for implementation research, the rapid pace of scale up which often precludes comparison areas, and the fact that "exposure" to CHWs or intensity of the programme is difficult to assess. Programmes typically contain multiple complex interventions (e.g. integrated community case management) which makes it difficult to disentangle the impact of individual elements. Programmes may operate in unique constellations of context (e.g. health systems and broader historical social, political, economic, and geographic contexts), making interpretation and generalizability difficult. Furthermore, the quality of data captured on community level activities is often poor and incomplete. This situation has led to very few rigorous experimental research studies but there has also been little attention paid to contextmechanism-outcome research questions, including implementation research. 
Evidence for impact of CHW programmes on maternal, newborn and child health When taking a broad view of community-based programmes, inclusive of CHW programmes focusing on child health, there is evidence for impact on child mortality through communitybased treatment of pneumonia, ${ }^{12}$ malaria ${ }^{13,14}$ and neonatal sepsis. ${ }^{15}$ Recent systematic reviews have concluded that community-based interventions and strategies can improve maternal and newborn outcomes, including morbidity and mortality of women, mortality and morbidity of neonates, and care-related outcomes in low and middle-income countries (LMICs). ${ }^{15-17}$

With regard to child health outcomes, evidence exists for positive impacts of CHWs on immunization uptake ${ }^{9}$, breastfeeding ${ }^{9}$, morbidity and mortality, ${ }^{18}$ care-seeking at health facilities for sick young infants ${ }^{19}$; and prompt treatment of fever with appropriate antimalarials. $^{20} \mathrm{~A}$ recent assessment of success factors for reducing maternal and child mortality in 10 Millennium Development Goal fast-track countries, identified the scale up of community-based health care as a key success factor in Ethiopia, Nepal and Rwanda. ${ }^{21}$

There is also substantial evidence for the impact of antenatal and postnatal home visits by CHWs on neonatal mortality, which now constitutes $45 \%$ of under- 5 deaths globally. ${ }^{2}$ A metaanalysis of nine trials in Africa and Asia reported a 21\% reduction in neonatal mortality due to antenatal and postnatal home visit interventions. ${ }^{22}$

Another community-based approach for which evidence is accumulating is women's groups, often facilitated by a female CHW. A systematic review and meta-analysis of seven trials of women's groups practicing participatory learning and action found that when at least $30 \%$ of pregnant women participated in the groups, run by local female facilitators, there was a $49 \%$ reduction in maternal mortality and a 33\% reduction in neonatal mortality. ${ }^{17}$ Whilst these women's group interventions hold much promise of impact, they have yet to be taken to scale within LMICs.

Besides proximal interventions for child health, in some countries the package of communitybased services also includes early child development. In Pakistan, the "lady health workers'” home visit package includes counselling mothers on responsive stimulation. A cluster randomised trial of this intervention reported significantly higher net development scores on cognitive, language, and motor scales at 12 and 24 months of age, and on a social-emotional scale at 12 months of age. ${ }^{23}$ 
The Philani Maternal Child Health and Nutrition Project in an urban settlement in Cape Town, South Africa, also reported positive effects of a mentor mother home visit programme, including training on responsive stimulation, on underweight and on severe underweight amongst children 0-6 years old. ${ }^{24}$

Besides evidence of impact, there is also evidence of quality of care delivered by CHWs. Research undertaken in Bangladesh found that CHWs can effectively screen (using mid-upper arm circumference) and treat uncomplicated cases of severe acute malnutrition (SAM) with ready to use therapeutic food (RUTF), showing a high degree of compliance with case management guidelines. ${ }^{25}$ A study conducted amongst health extension workers (HEWs) in Ethiopia included observation of consultations with sick children and gold standard reexaminations. The study found that HEWs were able to correctly manage $72 \%$, $79 \%$ and $59 \%$ of children with pneumonia, diarrhoea and malnutrition respectively. ${ }^{26}$

A recent survey in Kenya was conducted amongst caregivers of children under-5 who had sought care for pneumonia from either a CHW or a facility-based health worker. The study found that caregivers perceived CHWs to provide higher quality of care in terms of accessibility and patient relationships, compared to facility-based health workers and equal quality of care on clinical aspects. ${ }^{27}$

\section{Evidence for impact at scale}

There are examples of several countries with national CHWs programmes that have achieved large reductions in under-5 mortality and improvements in nutritional status of children. These examples may hold many lessons for countries in the early stages of implementation.

Ethiopia has achieved remarkable declines in under-5 mortality. According to the 2015 UN Inter-Agency Group for Child Mortality Estimation (IGME) report, Ethiopia reached its target for Millennium Development Goal 4 for child survival with an estimated under-five mortality rate of 59 per 1000 live births in 2015, a decline from 205 in 1990. This represents an average reduction in mortality of $5 \%$ per year; higher than the average for sub-Saharan Africa $(2.9 \%)^{1}$ One of the largest contributors to this under-5 mortality reduction was a decrease in stunting prevalence amongst children under-5 from 67\% in 1992 to $40 \%$ in 2014. ${ }^{2,28}$ During this period, major policy and program activities related to child survival 
were initiated in Ethiopia which built on major reforms starting from the 1990s to decentralise and reorganise the health system. An ambitious Health Extension Programme was launched in 2003 which aimed to provide universal access to mainly preventive primary health care services, through more than 34000 locally recruited, government-salaried health extension workers (HEWs). Infrastructural investments were made to construct over 16,000 health posts where these HEWs provide services.

The country's first national nutrition strategy and national nutrition programme were launched in 2008 which included scale up of the community-based nutrition programme. ${ }^{28}$ An impact evaluation of the community-based nutrition programme in four regions, delivered by HEWs-- with a ratio of 1 HEW per 23 children--and community volunteers, found substantial changes in infant and young child feeding (increased exclusive breastfeeding) and reductions in underweight of $2 \mathrm{ppts} /$ year.$-^{29}$

Malawi was one of the first countries in sub-Saharan Africa to have achieved the MDG4 target with a reduction from 245 to 68 deaths per 1000 live births between 1990 and 2013 ${ }^{1}$. Malawi's CHWs (called health surveillance assistants, HSAs) have played a central role in the delivery of health services in Malawi since the 1960's, delivering an increasingly broad array of services at the community level ${ }^{30-32}$. Initially operating as environmental health outreach assistants concentrating on water and sanitation; since 1995 HSAs have been formally recruited and salaried by the Ministry of Health. In 2011 there were over 10,000 HSAs in the country of which 3800 had been trained to diagnose and treat children with malaria, pneumonia and diarrhea (known as integrated community case management, iCCM) ${ }^{31}$. Small-scale evaluations during the early period of iCCM implementation (2009-2011) revealed high demand for HSA services ${ }^{31}$ and quality of care similar to that provided by nurses in first-level facilities ${ }^{33}$. The additional coverage for child health services due to community-based delivery has contributed to the large decline in child mortality in Malawi. ${ }^{34}$

Brazil is another country which has achieved remarkable reductions in under-5 mortality and stunting. Stunting prevalence amongst children under 5 years declined from 37\% in 1974/75 to $7 \%$ in 2006/07. Regional differences in stunting and child mortality also decreased. ${ }^{35}$ Four contributory factors to the reductions in stunting were identified including both nutritionspecific and nutrition-sensitive interventions ${ }^{36}$, which included actions to address social determinants: increased maternal education, increased purchasing power in the poorest 
populations, a substantial expansion in coverage of maternal and child health services, and the expansion of public water supply and sewage services. ${ }^{35}$ Changes in these determinants could plausibly have promoted child growth by improving diets (breastfeeding, complementary feeding, and the diversity of the family diet), reducing infections (especially those that cause diarrhoea), and contributing to better child care. The expansion in coverage of maternal and child health services was largely brought about through two programmes: the national community health worker programme and the family health programme which extended access to the poorest areas of the country as well as a large social protection initiative through conditional cash transfers (Bolsa Familia). ${ }^{37}$

The scope of practice of CHWs (known as Community Health Agents) in Brazil includes conducting home visits, promotion of healthy practices, linking families to health services, expanded vaccination coverage, promotion of breastfeeding, increased use of oral rehydration salts, management of pneumonia and growth monitoring. ${ }^{38}$

\section{Key considerations for CHW programmes}

CHW programmes are commonly seen as a cheap solution to increase access to health services in contexts with inadequate funds available for health care, weak health systems and insufficient human resources for health. ${ }^{8}$ The failure to consider and address the multitude of factors at both a national and international level that influence the impact and sustainability of these programmes is a common reason for their failure (Figure 1). 
Figure 1: Factors influencing the success of CHW programmes

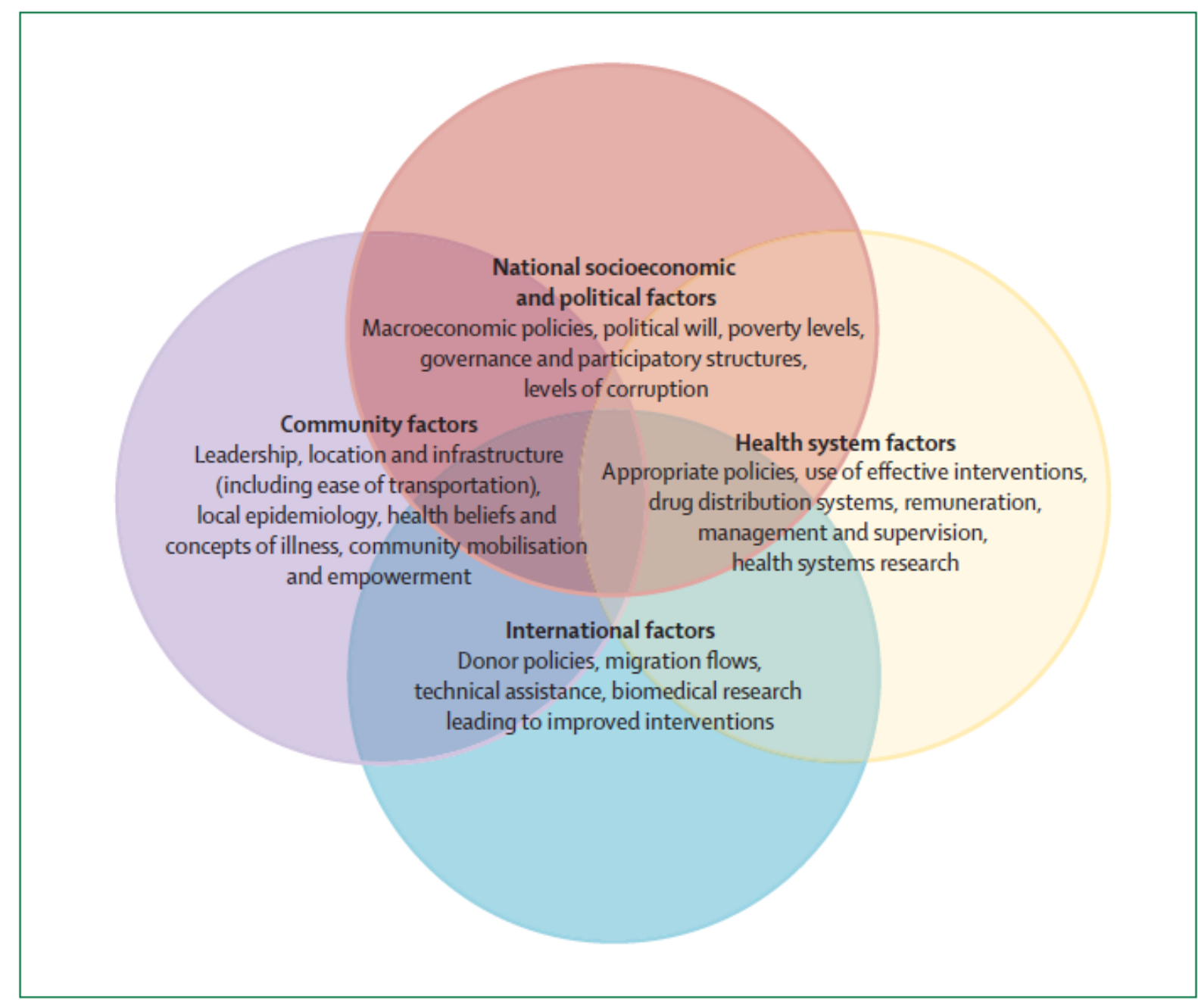

Reproduced with permission from: Haines et al. Lancet $2007 .^{8}$

At the international level, changing donor priorities and short-term funding cycles greatly influence the impact of country programmes. CHW programmes in most low income countries are heavily donor dependent ${ }^{39}$ for major components including $\mathrm{CHW}$ training and $\mathrm{CHW}$ salaries or stipends. Few countries incorporate CHWs as formally salaried members of the health workforce (with the usual labour rights). ${ }^{39}$ This situation impacts on the demand for services from communities ${ }^{40}$ when programmes are seen as transitory and vulnerable and almost certainly negatively affects motivation of CHWs. ${ }^{41,42}$ Furthermore, the donor dependent nature of CHW programmes has often resulted in the reinforcement of vertical approaches with donors paying for disease-specific CHWs to treat malaria or provide adherence support for antiretrovirals or TB treatment rather than for comprehensive, integrated programmes. ${ }^{43}$ 
At the national level, high level commitment and prioritization of $\mathrm{CHW}$ programmes by national governments, endorsement in policy documents (e.g. the national nutrition or health strategy) and commitment of domestic resources contribute positively to programme impact and sustainability. Rwanda, Brazil, Malawi and Ethiopia are all examples of countries which have scaled up community-based programmes focusing strongly on child health. Key features of these programmes include government ownership and incorporation of CHWs into the human resources for health wage bill.

The ratio of CHWs to population is also an important factor in impact which has been shown in relation to nutrition specific outcomes in Ethiopia. ${ }^{29}$ Optimal ratios of CHWs to population should be country-specific, taking into account the burden of disease (e.g. using under 5 mortality as an indicator) and epidemiological profile, CHW status as volunteer or fulltime equivalent, population density, geographic location, health infrastructure, road and transport infrastructure.

Related to the issue of ratios is the level and scope of practice of CHWs. In several countries, most notably Ethiopia, Niger and Mali, the community-based delivery platform consists of two tiers of workers, a lower volunteer cadre (known as the health development army in Ethiopia and relais communautaire in Mali and Niger) who are mainly responsible for promoting key family practices including breastfeeding, newborn care, hygiene and sanitation. ${ }^{44}$ This level is supported by a higher level of CHW who tends to have more education, is trained for longer, salaried, based in a fixed health post and whose scope also includes curative functions such as treatment of children with malaria, pneumonia, diarrhoea, severe acute malnutrition and in Ethiopia, newborn sepsis. ${ }^{44}$ This split in roles and tasks promotes better coverage, as ratios of volunteers to population are much higher than for the formal CHW level and this ensures that many preventive and promotive activities are undertaken at household level and are not neglected with the burden of curative tasks. ${ }^{44}$ In contexts where there is a high acceptance of volunteerism among both communities and CHWs, such as in Nepal, this may be an appropriate approach; however the volunteers still require supervision, kits and supplies etc. which would impact on cost, and the availability of the volunteers may be limited by their own family demands and livelihood activities. ${ }^{45}$ Due consideration should be given to how volunteer programmes may reinforce gender norms that hinder women's opportunities for earning money and advancing their careers. In some contexts, volunteers receive lunch allowances or other reimbursements at high enough levels that they are essentially working in positions which, 
though perhaps giving them high status, are actually very low-paid jobs. There is a resulting problem with high turnover-and thus the need for substantial ongoing recruitment and training--as they find better paid work.

Basic information on the background characteristics, training, and spatial distribution of CHWs is essential for effective planning, human resource management, supply chain management, emergency preparedness and response, monitoring, evaluation and research - indeed all aspects of health systems development. National georeferenced censuses of CHWs in Liberia, Malawi, Niger and Sierra Leone have underscored the importance of this information. ${ }^{46} \mathrm{CHW}$ background characteristics (e.g. gender), training, ratios of CHWs per population, and contributions of CHWs to additional geographic access to services tended to vary across space (Oliphant, to be published). For instance, most CHWs in Sierra Leone were male, except near urban areas where there were significant concentrations of female CHWs - indicating a spatialCHW gender association perhaps due to the volunteer nature of CHW work in Sierra Leone, as female CHWs reported being engaged in petty trade as their main livelihood and males reported farming as their main livelihood (Oliphant, to be published). Depending on the intervention, CHWs (called community health volunteers or CHVs) in Liberia provided an additional $10 \%-20 \%$ of the population geographic access to services (beyond the population covered within $5 \mathrm{~km}$ of a health facility) (Oliphant, to be published).

A recent systematic review of programmatic factors effecting CHW performance ${ }^{47}$ concluded that higher education, personal experience with the health condition of their clients, having fewer household duties, and being dependent on income generated from CHW activities were positively associated with CHW performance. Gender, community of origin, age, marital status and social class had mixed effects on CHW performance. For instance, female CHWs have been found to perform better than male CHWs in promotion of maternal health, follow up on antiretroviral therapy and tuberculosis treatment, while male CHWs have been found to perform better on record keeping. Countries should consider how their criteria for CHW selection may impact CHW performance, and how their choice of criteria may reinforce or challenge prevailing gender dynamics and social norms. Countries should also consider spatialsocial/economic dynamics in programme planning as illustrated by the examples from Liberia and Sierra Leone. 
Community trust and demand for services is a key factor in the success of CHW programmes. Research suggests that community participation in the selection and monitoring of CHWs is critical $^{8,48}$ and structures such as village health committees can play an important advocacy and social mobilisation role through their interaction with CHWs. ${ }^{49}$ Low demand for community services could be due to lack of trust in CHW skills, low availability of CHWs or cultural beliefs, particularly in the context of newborn interventions. ${ }^{26}$

There are several research priorities in the area of community-based health care delivery which deserve attention. Much of the existing evidence base comes from small scale research studies or pilot projects. There is a need for more evidence of impact at scale which will require the availability of substantive funding, innovative research designs based on plausibility methods. ${ }^{50}$ Embedded implementation research also holds promise as an approach to increasing ownership of research and involving key decision-makers and stakeholders throughout the process. $^{51}$

There is a clear gap in the evidence for the optimal scope of practice for CHWs. In the context of the triple burden of longstanding undernutrition and communicable diseases and newer emergence of non-communicable diseases occurring in low and middle-income countries, CHWs will increasingly need to include the management of chronic non-communicable diseases and prevention of overweight/obesity in their scope which will add to the already large workload of CHWs. This will have implications for ratios and skill levels. Operational research to understand the interactions between scope of practice and population ratios is greatly needed to assist countries with CHW planning and scale up. Research is also required to better understand the potential role of CHWs in affecting the social determinants of health within households and communities.

\section{Conclusions}

In the current SDG era, much remains to be achieved in terms of improving the health of children and reducing the large disparities between and within regions. Many of the high impact interventions can and should be delivered through community-based delivery platforms and there is accumulating evidence of the effectiveness of CHWs in delivering many preventive, promotive and curative child health services; however the shift in these tasks from facility to community level requires careful consideration of community participation in selection and ongoing monitoring, optimal ratios of CHWs to population, the spatial distribution of CHWs 
vis a vis need, the roles of CHWs and where applicable, volunteer cadres and the balance in scope to ensure that preventive, promotive and curative tasks are included in the package of care. Furthermore, health system support in the form of ongoing training and supervision, reliable systems for supplies and commodities and robust community information systems play a critical role.

The World Health Organisation is in the process of developing guidelines on health policy and system support to optimize community health worker programmes which will help to address a vacuum in guidance on important aspects of CHW programmes. Attention to domestic prioritization, support and financing of community delivery systems is critical to prevent these programmes from becoming another 'magic bullet'. 
1. UNICEF. Levels and Trends in Child Mortality: Report 2015. New York: UNICEF, 2015.

2. UNICEF. Countdown to 2015 maternal newborn and child survival: A Decade of Tracking Progress for Maternal, Newborn and Child Survival: The 2015 Report. New York: UNICEF, 2015.

3. Amouzou A, Kozuki N, Gwatkin DR. Where is the gap?: the contribution of disparities within developing countries to global inequalities in under-five mortality. BMC Public Health 2014; 14(1): 216. http://dx.doi.org/10.1186/1471-2458-14-216.

4. WHO. Declaration of Alma-Ata. Alma Ata: WHO, 1978.

5. Gilson L, Walt G, Heggenhougen K, et al. National community health worker programs: how can they be strengthened? J Public Health Policy 1989; 10(4): 518-32. https://www.ncbi.nlm.nih.gov/pubmed/2621254.

6. Schneider H, Okello D, Lehmann U. The global pendulum swing towards community health workers in low- and middle-income countries: a scoping review of trends, geographical distribution and programmatic orientations, 2005 to 2014. Hum Resour Health 2016; 14(1): 65. https://www.ncbi.nlm.nih.gov/pubmed/27784298.

7. Lehmann U, Sanders D. Community health workers: What do we know about them? The state of the evidence on programmes, activities, costs and impact on health outcomes of using community health workers. Geneva: World Health Organisation, 2007.

8. Haines A, Sanders D, Lehmann U, et al. Achieving child survival goals: potential contribution of community health workers. The Lancet 2007; 369(9579): 2121-31. http://www.sciencedirect.com/science/article/pii/S0140673607603250.

9. Lewin S, Munabi-Babigumira S, Glenton C, Daniels K, Bosch-Capblanch X, Wyk BE. Lay health workers in primary and community health care for maternal and child health and the management of infectious diseases. Cochrane Database Syst Rev 2010; 3.

10. Mwai GW, Mburu G, Torpey K, Frost P, Ford N, Seeley J. Role and outcomes of community health workers in HIV care in sub-Saharan Africa: a systematic review. Journal of the International AIDS Society 2013; 16(1): 18586.

http://www.ncbi.nlm.nih.gov/pmc/articles/PMC3772323/.

11. Lassi ZS, Bhutta ZA. Community-based intervention packages for reducing maternal and neonatal morbidity and mortality and improving neonatal outcomes. Cochrane Database Syst Rev 2015; 3.

12. Sazawal S, Black RE. Effect of pneumonia case management on mortality in neonates, infants, and preschool children: a meta-analysis of community-based trials. Lancet Infect Dis 2003; 3(9): 547-56.

http://www.ncbi.nlm.nih.gov/entrez/query.fcgi?cmd=Retrieve\&db=PubMed\&dopt=Citation \&list_uids=12954560.

13. Yeboah-Antwi K, Pilingana P, Macleod WB, et al. Community case management of fever due to malaria and pneumonia in children under five in Zambia: a cluster randomized controlled trial. PLoS Medicine 2010; 7(9): 1139.

14. Chinbuah MA, Kager PA, Abbey M, et al. Impact of community management of fever (using antimalarials with or without antibiotics) on childhood mortality: a clusterrandomized controlled trial in Ghana. The American journal of tropical medicine and hygiene 2012; 87(5 Suppl): 11-20. http://www.ncbi.nlm.nih.gov/pubmed/23136273.

15. Lassi ZS, Bhutta ZA. Community-based intervention packages for reducing maternal and neonatal morbidity and mortality and improving neonatal outcomes. Cochrane Database Syst Rev 2015; (3): CD007754. 
http://www.ncbi.nlm.nih.gov/entrez/query.fcgi?cmd=Retrieve\&db=PubMed\&dopt=Citation \&list_uids=21069697.

16. Lassi ZS, Middleton PF, Bhutta ZA, Crowther C. Strategies for improving health care seeking for maternal and newborn illnesses in low- and middle-income countries: a systematic review and meta-analysis. 2016 2016; 9.

http://www.globalhealthaction.net/index.php/gha/article/view/31408.

17. Prost A, Colbourn T, Seward N, et al. Women's groups practising participatory learning and action to improve maternal and newborn health in low-resource settings: a systematic review and meta-analysis. The Lancet 2013; 381(9879): 1736-46.

http://dx.doi.org/10.1016/S0140-6736(13)60685-6.

18. Lewin S, Munabi-Babigumira S, Glenton C, et al. Lay health workers in primary and community health care for maternal and child health and the management of infectious diseases. Cochrane Database Syst Rev 2010; (3): CD004015.

http://www.ncbi.nlm.nih.gov/pubmed/20238326.

19. Tripathi A, Kabra SK, Sachdev HP, Lodha R. Home visits by community health workers to improve identification of serious illness and care seeking in newborns and young infants from low- and middle-income countries. J Perinatol 2016; 36 Suppl 1: S74-82.

20. Okwundu CI, Nagpal S, Musekiwa A, Sinclair D. Home- or community-based programmes for treating malaria. Cochrane Database of Systematic Reviews 2013; (5). http://dx.doi.org/10.1002/14651858.CD009527.pub2.

21. Kuruvilla S, Schweitzer J, Bishai D, et al. Success factors for reducing maternal and child mortality. Bulletin of the World Health Organization 2014; 92(7): 533-44B. http://www.ncbi.nlm.nih.gov/pubmed/25110379.

22. Kirkwood BR, Manu A, ten Asbroek AH, et al. Effect of the Newhints home-visits intervention on neonatal mortality rate and care practices in Ghana: a cluster randomised controlled trial. Lancet 2013; 381(9884): 2184-92.

http://www.ncbi.nlm.nih.gov/pubmed/23578528.

23. Yousafzai AK, Rasheed MA, Rizvi A, Armstrong R, Bhutta ZA. Effect of integrated responsive stimulation and nutrition interventions in the Lady Health Worker programme in Pakistan on child development, growth, and health outcomes: a cluster-randomised factorial effectiveness trial. The Lancet 2014; 384(9950): 1282-93. http://dx.doi.org/10.1016/S01406736(14)60455-4.

24. Tomlinson M, Hartley M, Le Roux IM, Rotheram-Borus MJ. The Philani Mentor Mothers Intervention: neighbourhood wide impact on child growth in Cape Town's periurban settlements. Vulnerable Children and Youth Studies 2016; 11(3): 211-20.

http://dx.doi.org/10.1080/17450128.2016.1214770.

25. Puett C, Coates J, Alderman H, Sadler K. Quality of care for severe acute malnutrition delivered by community health workers in southern Bangladesh. Matern Child Nutr 2013; 9(1): 130-42.

26. Miller NP, Amouzou A, Tafesse M, et al. Integrated Community Case Management of Childhood Illness in Ethiopia: Implementation Strength and Quality of Care. The

American journal of tropical medicine and hygiene 2014. http://www.ncbi.nlm.nih.gov/pubmed/24799369.

27. Shaw BI, Asadhi E, Owuor K, et al. Perceived Quality of Care of Community Health Worker and Facility-Based Health Worker Management of Pneumonia in Children Under 5 Years in Western Kenya: A Cross-Sectional Multidimensional Study. The American journal of tropical medicine and hygiene 2016; 94(5): 1170-6.

https://www.ncbi.nlm.nih.gov/pubmed/26976883.

28. Doherty T, Rohde S, Besada D, et al. Reduction in child mortality in Ethiopia: analysis of data from demographic and health surveys. Journal of global health 2016; 6(2). 
29. White J, Mason J. Assessing the impact on child nutrition of the Ethiopia communitybased nutrition programme. New Orleans: Tulane University, 2012.

30. Kadzandira J, Chilowa W. The role of Health Surveillance Assistants (HSAs) in the delivery of health services and immunisation in Malawi. Lilongwe: Malawi Ministry of Health and UNICEF, 2001.

31. Nsona H, Mtimuni A, Daelmans B, et al. Scaling up integrated community case management of childhood illness: update from Malawi. The American journal of tropical medicine and hygiene 2012; 87(5 Suppl): 54-60.

http://www.ncbi.nlm.nih.gov/pubmed/23136278.

32. Smith S, Deveridge A, Berman J, et al. Task-shifting and prioritization: a situational analysis examining the role and experiences of community health workers in Malawi. Hum Resour Health 2014; 12: 24. http://www.ncbi.nlm.nih.gov/pubmed/24885454.

33. Gilroy KE, Callaghan-Koru JA, Cardemil CV, et al. Quality of sick child care delivered by Health Surveillance Assistants in Malawi. Health Policy Plan 2013; 28(6): 57385. http://www.ncbi.nlm.nih.gov/pubmed/23065598.

34. Doherty T, Zembe W, Ngandu N, et al. Assessment of Malawi's success in child mortality reduction through the lens of the Catalytic Initiative Integrated Health Systems Strengthening programme: Retrospective evaluation. Journal of global health 2015; 5(2): 020412.

35. Victora CG, Aquino EM, do Carmo Leal M, Monteiro CA, Barros FC, Szwarcwald CL. Maternal and child health in Brazil: progress and challenges. Lancet; 377(9780): 186376.

http://www.ncbi.nlm.nih.gov/entrez/query.fcgi?cmd=Retrieve\&db=PubMed\&dopt=Citation \&list_uids=21561656.

36. Ruel MT, Alderman H. Nutrition-sensitive interventions and programmes: how can they help to accelerate progress in improving maternal and child nutrition? The Lancet 2013; 382(9891): 536-51. http://dx.doi.org/10.1016/S0140-6736(13)60843-0.

37. Paes-Sousa R, Pacheco-Santos LM, E. Effects of a conditional cash transfer programme on child nutrition in Brazil. Bulletin World Health Organ 2011 2011; 89: 496503.

38. Bhutta ZA, Lassi ZS, Pariyo G, Huicho L. Global Experience of Community Health Workers for Delivery of Health Related Millennium Development Goals: A Systematic Review, Country Case Studies, and Recommendations for Integration into National Health Systems. Geneva: World Health Organisation, 2010.

39. Rasanathan K, Muniz M, Bakshi S, et al. Community case management of childhood illness in sub-Saharan Africa - findings from a cross-sectional survey on policy and implementation. Journal of global health 2014; 4(2): 020401.

40. Daniels K, Sanders D, Daviaud E, Doherty T. Valuing and Sustaining (or Not) the Ability of Volunteer Community Health Workers to Deliver Integrated Community Case Management in Northern Ghana: A Qualitative Study. PloS one 2015; 10(6): e0126322.

41. Dil Y, Strachan D, Cairncross S, Korkor A, Hill Z. Motivations and challenges of community-based surveillance volunteers in the northern region of Ghana. J Commun Health 2012; 37: 1192 - 8.

42. Bhattacharyya K, Winch P, LeBan K, Tien M. Community Health Worker Incentives and Disincentives: How They Affect Motivation, Retention, and Sustainability. Virgian Basics II, 2001.

43. Tulenko K, Mogedal S, Afzal MM, et al. Community health workers for universal health-care coverage: from fragmentation to synergy. Bulletin of the World Health Organization 2013; 91(11): 847-52. https://www.ncbi.nlm.nih.gov/pubmed/24347709. 
44. Leon N, Sanders D, Van Damme W, et al. The role of 'hidden' community volunteers in community-based health service delivery platforms: examples from sub-Saharan Africa. Global health action 2015; 8: 27214. http://www.ncbi.nlm.nih.gov/pubmed/25770090. 45. Daniels K, Sanders D, Daviaud E, Doherty T. Valuing and Sustaining (or Not) the Ability of Volunteer Community Health Workers to Deliver Integrated Community Case Management in Northern Ghana: A Qualitative Study. PloS one 2015; 10. http://dx.doi.org/10.1371/journal.pone.0126322.

46. Oliphant NP. Putting CHWs on the map: Toward a geography of national CHW programs implementing integrated community case management (iCCM): PhD protocol. Cape Town: University of the Western Cape; 2015.

47. Kok MC, Dieleman M, Taegtmeyer M, et al. Which intervention design factors influence performance of community health workers in low- and middle-income countries? A systematic review. Health Policy Plan 2014.

http://www.ncbi.nlm.nih.gov/pubmed/25500559.

48. Sanders D. Success Factors in Community-Based Nutrition Programmes. Food and nutrition bulletin 1999; 20(3): 307-14. http://fnb.sagepub.com/content/20/3/307.abstract. 49. Zembe-Mkabile WZ, Jackson D, Sanders D, et al. The 'community' in community case management of childhood illnesses in Malawi. 2016 2016; 9.

http://www.globalhealthaction.net/index.php/gha/article/view/29177.

50. Habicht JP, Victora CG, Vaughan JP. Evaluation designs for adequacy, plausibility and probability of public health programme performance and impact. International journal of epidemiology 1999; 28(1): 10-8.

51. de Savigny D, Taghreed A, Alliance for Health Policy and Systems Research, World Health Organization. Systems Thinking for Health Systems Strengthening. Geneva: WHO, 2009. 